http://jmscr.igmpublication.org/home/

ISSN (e)-2347-176x ISSN (p) 2455-0450

crossref DOI: https://dx.doi.org/10.18535/jmscr/v9i7.24

Journal Of Medical Science And Clinical Research

\title{
Yellow Oleander seed poisoning induced expression of Brugada phenocopy in Electrocardiogram - A rare case report
}

\author{
Authors \\ Dr Bigyan Keshari Kapardar ${ }^{1}$, Dr Sourabh Pradhan ${ }^{2}$ \\ ${ }^{1,2}$ Senior Resident, Department of General Medicine, All India Institute of Medical Sciences, Bhubaneswar, \\ India \\ Corresponding Author
}

Dr Bigyan K. Kapardar

\begin{abstract}
Brugada phenocopy is a new electrocardiogram phenomenon which is a Brugada-like pattern in ECG which has varied etiologies. It differs from true Brugada's syndrome in lacking the presence of underlying inherited channelopathy. Also the etiologies are reversible conditions. Once the reversible condition resolves, the Brugada phenocopy on ECG also disappears. Here we report a rare case of Brugada phenocopy as a covert expression of Yellow Oleander (Thevetia peruviana) seeds poisoning.
\end{abstract}

\section{Introduction}

Yellow oleander (Thevetia peruviana) is an ornamental tree commonly found in tropics and subtropics. Consumption of yellow oleander seeds as a method of committing suicide is very common in South East Asia. It is used for suicide, homicide and for abortion in India and other parts of world ${ }^{[1]}$. Worldwide accidental poisoning with yellow oleander is very common in children due to the fascinating colour and shape of its seeds and leaves ${ }^{[1]}$. All parts of the plant are poisonous. Commonly poisoning occurs following ingestion of the seeds or the crushed/chewed fruit. The number of seeds needed to cause significant poisoning is very variable. The active principles are at least eight types of glycosides ${ }^{[2]}$ mainly thevetin, thevetoxin, cerebrin and nerifolin etc which act like digitalis on cardiac tissue. Brugada phenocopy is a mimic of brugada pattern which is produced by a variety of etiological conditions namely metabolic conditions (hyperkalemia, hypokalemia), mechanical compression, myocardial infarction, pulmonary embolism, ECG modulation etc described in this article later. Here we report a case of Brugada Phenocopy in a 50 Years old female who presented with Type1Brugada pattern on ECG after consumption of crushed seeds of Yellow Oleander which spontaneously reverts to normal sinus rhythm after 5 days of hospitalisation.

\section{Case Report}

A 50 years female presented to our trauma and emergency department with alleged history of consumption of three crushed seeds of yellow oleander. She was initially taken to the nearest local district hospital where after giving stomach wash she was referred to our tertiary care hospital for further management. She was triaged as Emergency Severity Index level-3 (ESI-3) in our 
emergency department and kept in the red zone of triage. She was consciousand oriented to time, place and person. She was hemodynamically stable at the time of presentation to our emergency department. Her vitals measurement were respiratory rate of 18 per minute, Oxygen saturation of $99 \%$ at room air, heart rate of 96 beats per minute, regular and of normal volume and Blood pressure of $110 / 70 \mathrm{~mm}$ of $\mathrm{Hg}$ and without any external injury marks. However her initial Electrocardiogram showed sinus rhythm with HR- 92 per minute, PR interval of $203 \mathrm{mS}$ with Type-1 Brugada's pattern in the form of ST elevation of more than $2 \mathrm{~mm}$ in lead V2, Tinversion in V1-V4 and a QT/QTc of 336/417 mS as shown in figure-1. Her Troponin test was negative at that time. Her immediate ABG test ruled out the possibility of hyperkalemia or any other dyselectrolytemia. The patient did not have any history of palpitation or chest pain or any syncope history prior to this incident. Her family history did not suggest any similar complaints. She was kept under continuous cardiac monitoring in view of any impending cardiac toxicity with serial Troponin test being negative every 6 hourly. Her emergency 2D-ECHO was also came out to be normal study without any resting wall motion abnormality and showing good LV systolic function. After about 24 hours from the time of alleged consumption of oleander seeds she was hemodynamically stable and without any symptoms of chest pain or palpitation. Her repeat ECG on Day-2 of hospitalization showed sinus bradycardia with heart rate of 60 per minute, PR interval of $234 \mathrm{mS}$ with Type-2 Brugada's pattern in the form of Saddleback ST segment elevation of less than $2 \mathrm{~mm}$ and $\mathrm{T}$ wave inversion in leads V1-V3 and QT/QTc of 384/376 mS as shown in figure-2. Her routine investigations like Complete hemogram, Renal Function test, Liver function test with Serum electrolytes came out to be within normal limits. She was managed conservatively on symptomatic basis with daily repeat ECG testing and continuous hemodynamic monitoring. Her repeat ECG (Figure-3) till day-4 of hospitalisation showed type-2 Brugada's pattern without manifestation of any systemic cardiac symptoms. However at the time of discharge on day-5 the patient's Brugada pattern disappeared and ECG (figure-4) showed sinus rhythm with heart rate of 66 beats per minute and QT/QTc of $347 / 365 \mathrm{mS}$ and leads V2-V3 showed normal ST segment and $\mathrm{T}$ - wave.

As no provocative tests were performed in this case we report it as Brugada phenocopy class-B.

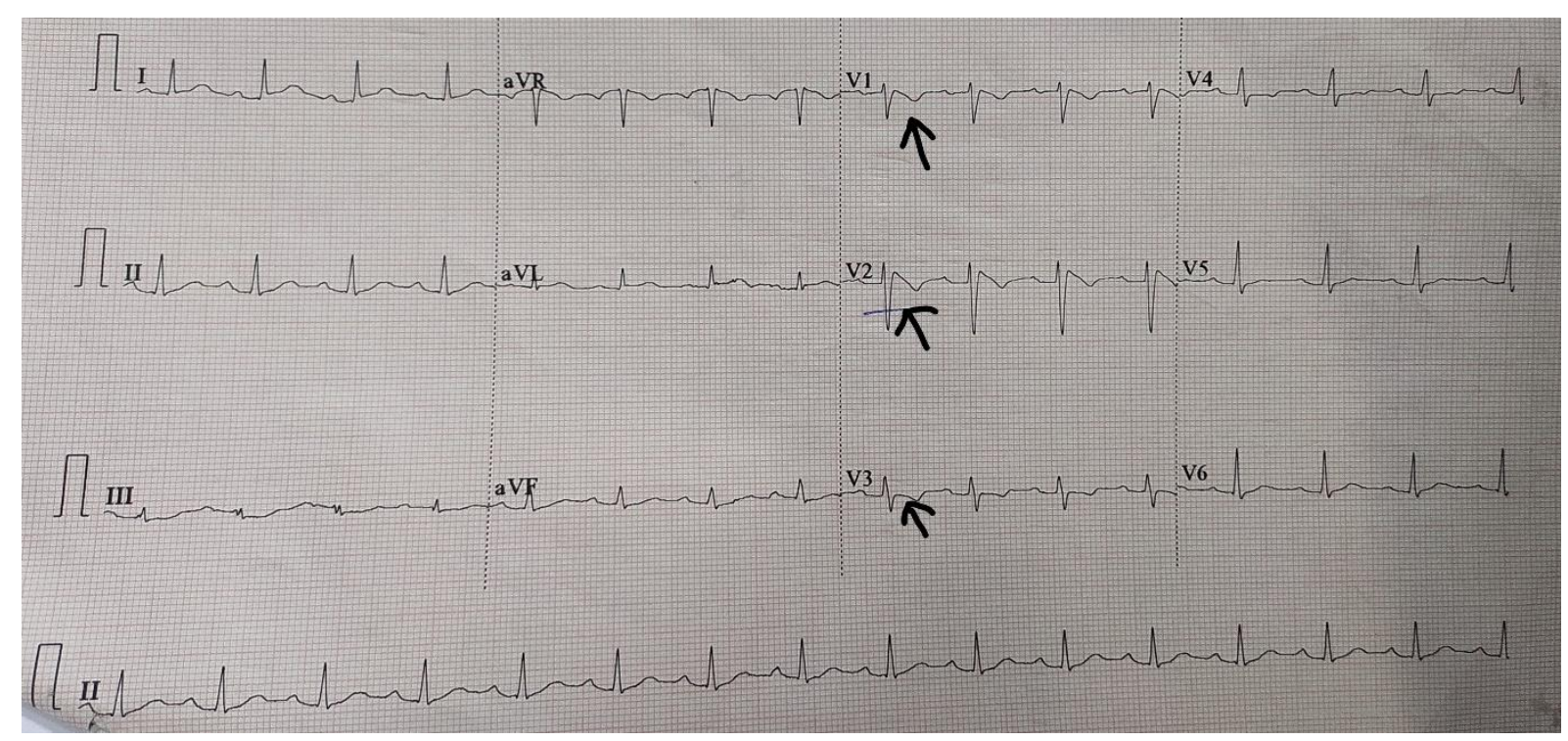

Figure-1 - showing Type-1 Brugada phenocopy on Day-1 


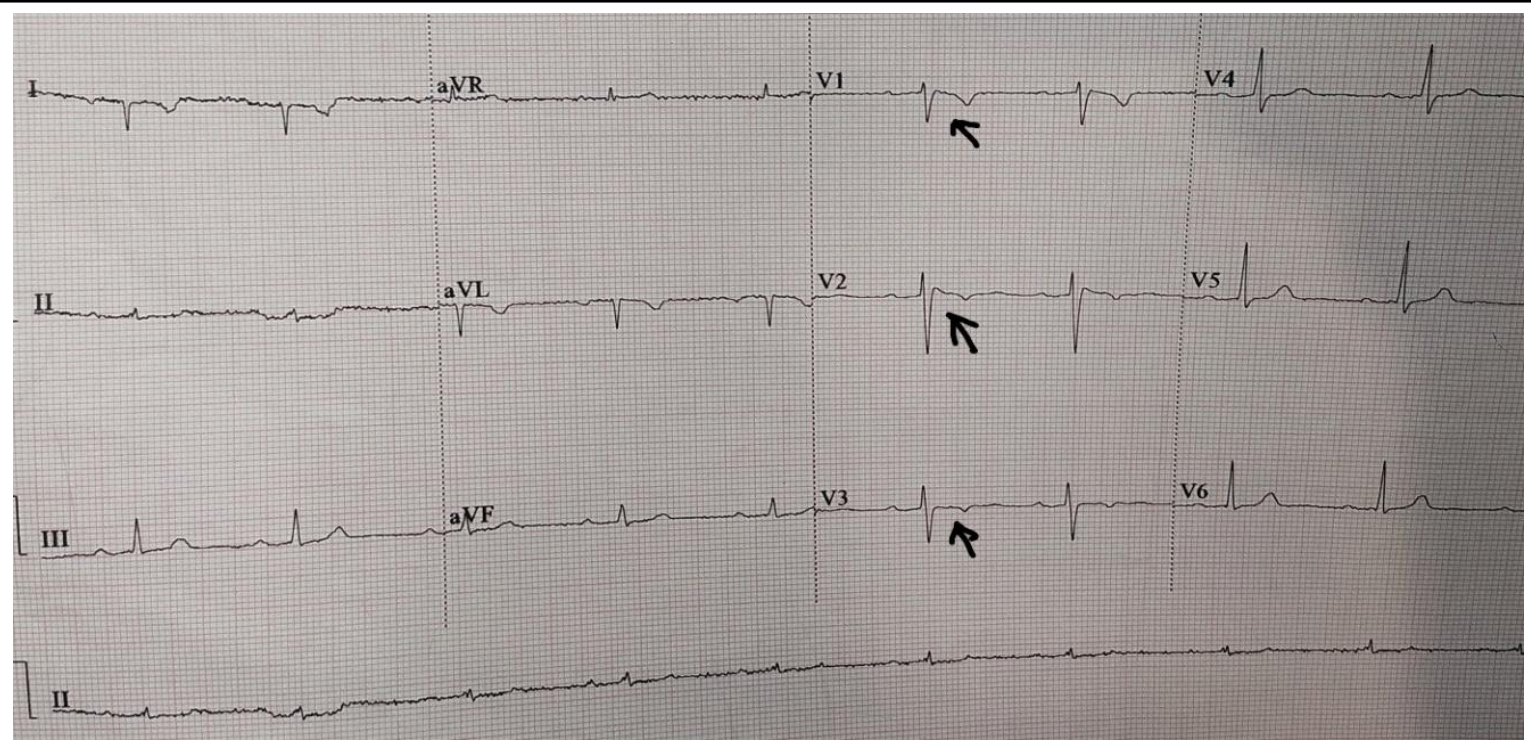

Figure-2 - showing Type-2 Brugada Phenocopy on day-2

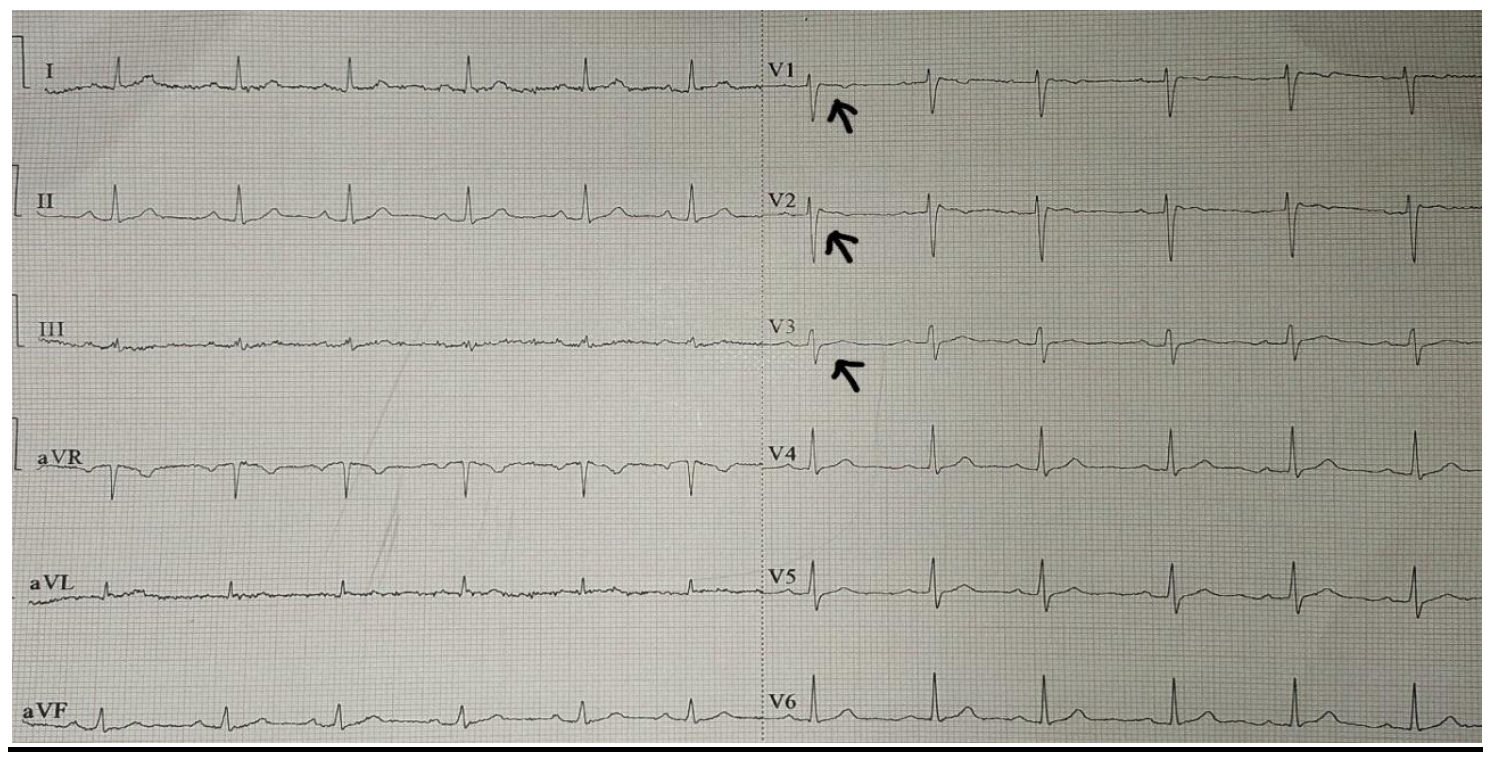

Figure-3 - showing Type-2 Brugada phenocopy on day-4

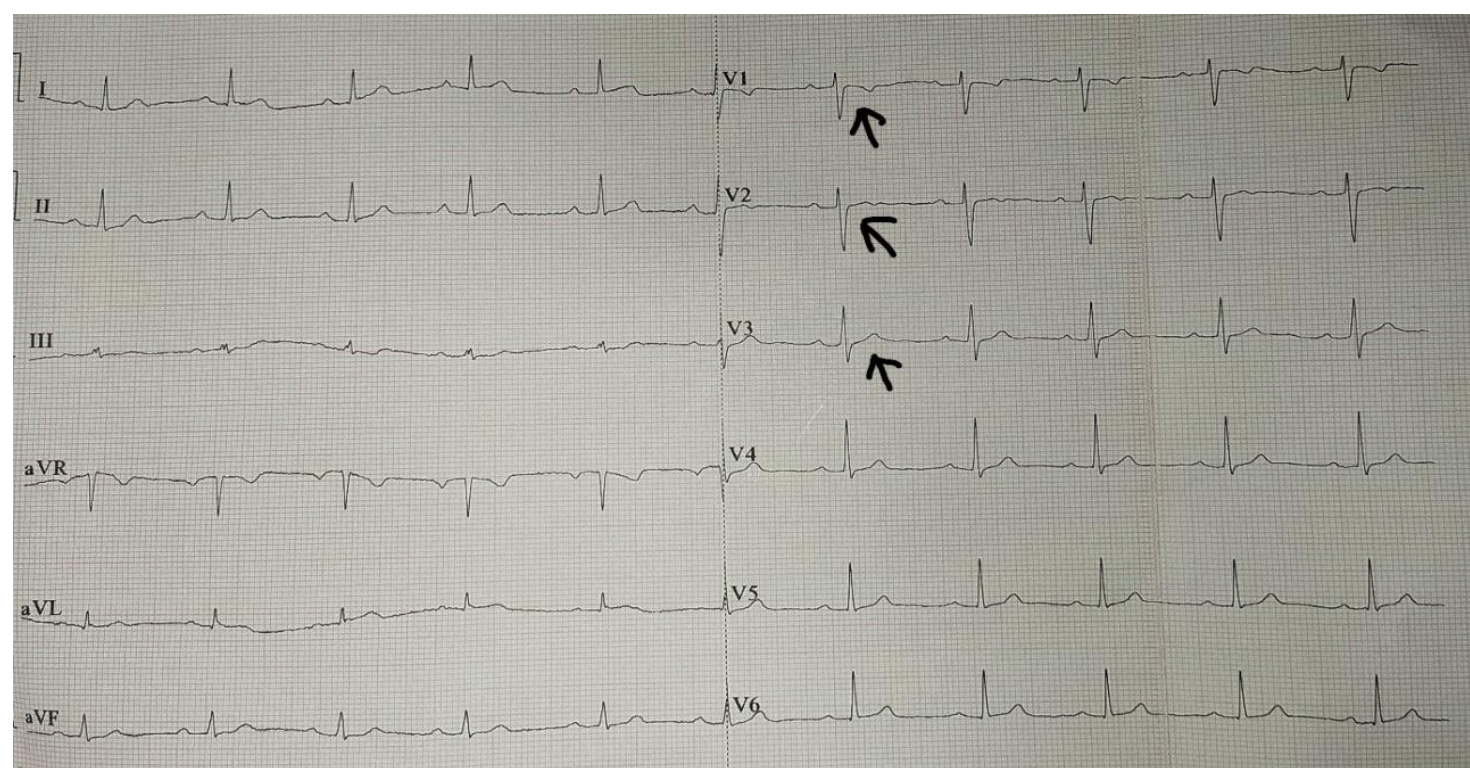

Figure-4- showing Normal sinus rhythm on day-5 (day of discharge) 


\section{Discussion}

Brugada syndrome accounts for $4-12 \%$ of all causes of sudden cardiac death in young adults ${ }^{[3]}$. The concept of Brugada phenocopies suggest ECG patterns characteristic of Brugadapattern that may appear and disappear in relation with multiple causes, but differ from Brugada syndrome in multiple aspects.

Depending on underlying mechanism the clinical conditions associated with Brugada Phenocopy on ECG can be broadly categorised into following six categories $^{[4-6]}$ like (1) Metabolic conditions ${ }^{[7-9]}$, (2) Mechanical compression, (3) Ischemia, (4) Myocardial and pericardial disease, (5) ECG modulation, and (6) Miscellaneous conditions like electrocution, neurological injuries, and surgical repair of congenital heart disease etc.

Brugada Phenocopy can be morphologically classified $^{[6]}$ into Type-1 and Type-2 depending on its resemblance to the ECG morphology of Brugada syndrome. It can be further classified into Class- A, B \& C depending upon the diagnostic criteria being fulfilled as follows. The consensus diagnostic criteria of Brugada Phenocopy have been adapted byAnselm DD et al in $2013^{[10]}$.

The occurance of Brugada pattern in our case can be discussed as follows:

\section{Toxicity due to consumption of seeds of Yellow oleander ;}

The seeds of Yellow oleander contains at least eight types of digoxin-like cardioactive steroids ${ }^{[2]}$ that includes Thevetin A and B, Peruvoside, Nerifolin, Thevetoxin, Ruvoside and theviridoside etc. The primary mechanism of action of these cardiac glycosides isinhabitation of $\mathrm{Na}^{+}-\mathrm{K}^{+}-$ ATPase channels causing intracellular retention of sodium. The retained intracellular sodium indirectly increases the concentration of intracellular calcium by decreasing intracellular calcium and release of more calcium from sarcoplasmic reticulum during systole. The resulting high intracellular calcium causes increased cardiac inotropy and bradycardia. All these mechanisms result in partial membrane depolarization that causes increased automaticity and ventricular ectopy. The resulting hyperkalemiadue to inhibition of $\mathrm{Na}^{+}-\mathrm{K}^{+}$-ATPase channels causes increases AV nodal block by hyperpolarizing cardiac conduction tissue ,that exacerbates conduction delays and ultimately bradydysrhythmias $^{[11]}$. Also the resulting decreased sodium current in the membrane during phase 3 and 4 of myocardial contraction may be an explanation for the Brugada phenocopy in such cases of poisoning by cardio-active steroids.

2. BrP due to use of inappropriate high pass filter in ECG ;

It has been already reported that use of inappropriate high pass filter can result in Brugada phenocopy ${ }^{[12]}$. But in our case all the ECGs were taken with the same high pass filter. All the four ECGs provided here have shown from Brugada phenocopy on the day of hospitalization to resolution to normal sinus rhythm during discharge of the patient. Hence it excludes as a possible cause in our case.

\section{Conclusion}

An international registry or database should be maintained to aim at furthering knowledge on etiologies, pathophysiology, and natural history of Brugada Phenocopy. It is unknown whether Brugada Phenocopy is a risk factor for malignant arrhythmias which can be managed if diagnosed on time. Further study is needed in cases with Brugada phenocopy induced by consumption of Yellow oleander to ascertain the long-term prognosis of these patients. Here we report that Brugada phenocopy can be induced by yellow oleander poisoning.

\section{References}

1. Eddleston M, Ariaratnam CA, Meyer PW. Epidemic of self-poisoning with seeds of the yellow oleander tree (Thevetia peruviana) in northern Sri Lanka. Trop Med Int Health 1999;4:266-73.DOI: 10.1046/j.1365-3156.1999.00397.x

2. Eddleston M, Ariaratnam CA, Sjöström L, Jayalath S, Rajakanthan K, Rajapakse S. 
Acute yellow oleander (Thevetia peruviana) poisoning: Cardiac arrhythmias, electrolyte disturbances and serum cardiac glycoside concentrations on presentation to hospital. Heart 2000;83: 301-6. DOI: 10.1136/heart.83.3.301

3. Sarquella-Brugada G, Campuzano O, Iglesias A, Sánchez-Malagón J, GuerraBalic M, Brugada J, et al. Genetics of sudden cardiac death in children and young athletes. Cardiol Young. 2013 Apr; 23(2):159-73.

DOI 10.1017/S1047951112001138

4. Baranchuk A, Nguyen T, Ryu MH, et al. Brugada phenocopy: new terminology and proposed classification. Ann NoninvasiveElectrocardiol 2012; 17: 299$314 . \quad$ DOI $\quad 10.1111 / \mathrm{j} .1542-$ 474X.2012.00525.x

5. Anselm DD, Gottschalk BH, Baranchuk A. Brugada phenocopies: consideration of morphologic criteria and early findings from an international registry. Can J Cardiol2014;30:1511-5.

DOI 10.1016/j.cjca.2014.09.023

6. Gottschalk B, Anselm DD, Baranchuk A. Brugada phenocopy: morphological classification and importance of provocative testing. Ann Noninvasive Electrocardiol 2014;19: 604-5. DOI 10.1111/anec. 12207

7. Hunuk A, Hunuk B, Kusken O, Onur OE. Brugada phenocopy induced by electrolyte disorder: a transient electrocardiographic sign. Ann Noninvasive Electrocardiol 2016;21:429-32. DOI 10.1111/anec. 12350

8. Genaro NR, Anselm DD, Cervino N, et al. Brugada phenocopy clinical reproducibility demonstrated by recurrent hypokalemia. Ann Noninvasive Electrocardiol 2014;19:387-90. DOI 10.1111/anec.12101

9. Meloche J, Gottschalk BH, Boles U, LaHaye S, Baranchuk A. Hypophosphatemia as a novel etiology of
Brugada phenocopy. Int J Cardiol 2016; 208:70-1.

DOI 10.1016/j.ijcard.2016.01.124

10. Anselm DD, Baranchuk A. Brugada phenocopy in the context of pulmonary embolism. Int J Cardiol2013;168:560. DOI 10.1016/j.ijcard.2013.01.169

11. Robert S. Hoffman, Mary Ann Howland, Neal A. Lewin, Lewis S. Nelson, Lewis R. Goldfrank: Goldfrank's Toxicologic Emergencies. 10th Edition, 1502-1509; 2015.

12. García-Niebla J, Serra-Autonell G. Bayés de Luna A. Brugada syndrome electrocardiographic pattern as a result of improper application of a high pass filter. Am J Cardiol. 2012 Jul 15;110(2):318-20. DOI 10.1016/j.amjcard.2012.04.038. 